\title{
An Updated genome annotation for the model marine bacterium Ruegeria pomeroyi DSS-3
}

\author{
Adam R Rivers, Christa B Smith and Mary Ann Moran*
}

\begin{abstract}
When the genome of Ruegeria pomeroyi DSS-3 was published in 2004, it represented the first sequence from a heterotrophic marine bacterium. Over the last ten years, the strain has become a valuable model for understanding the cycling of sulfur and carbon in the ocean. To ensure that this genome remains useful, we have updated 69 genes to incorporate functional annotations based on new experimental data, and improved the identification of 120 protein-coding regions based on proteomic and transcriptomic data. We review the progress made in understanding the biology of R. pomeroyi DSS-3 and list the changes made to the genome.
\end{abstract}

Keywords: Roseobacter, DMSP

\section{Introduction}

Ruegeria pomeroyi DSS-3 is an important model organism in studies of the physiology and ecology of marine bacteria [1]. It is a genetically tractable strain that has been essential for elucidating bacterial roles in the marine sulfur and carbon cycles [2,3] and the biology and genomics of the marine Roseobacter clade [4], a group that makes up $5-20 \%$ of bacteria in ocean surface waters $[5,6]$. Here we update the $R$. pomeroyi DSS-3 genome with 189 changes collected from the work of several research groups over the last ten years.

\section{Organism information}

Ruegeria pomeroyi DSS-3 (formerly Silicibacter pomeroyi DSS-3 [7]) is a motile gram-negative alphaproteobacterium in the marine Roseobacter lineage [8]. This mesophilic, heterotrophic bacterium was isolated from an estuary in coastal Georgia, U.S.A [9] (Table 1).

\section{Genome sequencing information Genome project history}

The genome of $R$. pomeroyi DSS-3 was sequenced in 2003 by The Institute for Genomic Research (now the J. Craig Venter Institute) using Sanger sequencing (Table 2), and was annotated using Glimmer 2 [20] and the TIGR Assembler [21]. The genome was published in 2004 [1].

\footnotetext{
* Correspondence: mmoran@uga.edu

Department of Marine Sciences, University of Georgia, Athens, GA, USA
}

\section{Genome properties}

The $R$. pomeroyi DSS-3 genome contains a 4,109,437 bp circular chromosome (5 bp shorter than previously reported [1]) and a 491,611 bp circular megaplasmid, with a $\mathrm{G}+\mathrm{C}$ content of 64.1 (Table 3). A detailed description of the genome is found in the original article [1].

\section{Reannotation}

The $R$. pomeroyi DSS-3 genome has been instrumental in expanding knowledge of the marine sulfur cycle, particularly the role of marine bacteria in controlling the flux of volatile sulfur to the atmosphere $[3,22]$ and the bacterial transformations of dimethylsulfoniopropionate (DMSP) [3,23], dimethylsulfide, and sulfonates [24,25]. Since 2006, many of the genes mediating the uptake and metabolism of DMSP have been identified from the $R$. pomeroyi DSS-3 genome. These include the demethylation pathway genes $d m d A B C D[2,22]$ and the cleavage pathway genes $d d d D, d d d P, d d d Q, d d d W, a c u K, a c u N$, $d d d A$ and $d d d C$ [23,26,27]. Although many genes were identified first in $R$. pomeroyi DSS-3, these are now known to be widespread in ocean surface waters and harbored by a number of other major marine bacterial taxa [28]. $R$. pomeroyi DSS-3 also transforms sulfonates and has served as a model for identifying genes required for the degradation of 2,3-dihydroxypropane-1-sufonate ( $h p s N O P$ ) [29], L-cysteate (cuyARZ) [30], taurine $(\operatorname{tau} X Y)$ and n-acetyltaurine (naaST) [24,31,32], 3-sulfolactate $(s l c D$, $\operatorname{suy} A B)[29,33]$ and isethionate (ise) [25]. 
Table 1 Classification and general features of Ruegeria pomeroyi DSS-3 according to MIGS recommendations [9]

\begin{tabular}{|c|c|c|c|}
\hline MIGS ID & Property & Term & Evidence code $^{\mathrm{a}}$ \\
\hline & Current classification & Domain Bacteria & TAS [10] \\
\hline & & Phylum Proteobacteria & TAS [11] \\
\hline & & Class Alphaproteobacteria & $\operatorname{TAS}[12,13]$ \\
\hline & & Order Rhodobacterales & TAS $[12,14]$ \\
\hline & & Family Rhodobacteraceae & $\operatorname{TAS}[12,15]$ \\
\hline & & Genus Ruegeria & TAS $[7,16-18]$ \\
\hline & & Species Ruegeria pomeroyi & $\operatorname{TAS}[7,19]$ \\
\hline & & Type strain DSS-3 = ATCC 700808 T= DSM 15171 & \\
\hline & Gram stain & Negative & TAS [8] \\
\hline & Cell shape & Rod & TAS [8] \\
\hline & Motility & Motile & TAS [8] \\
\hline & Sporulation & Non-sporulating & NAS \\
\hline & Temperature range & Mesophile $\left(10^{\circ} \mathrm{C}-40^{\circ} \mathrm{C}\right)$ & TAS [8] \\
\hline & Optimum temperature & $30^{\circ} \mathrm{C}$ & \\
\hline & Carbon source & $\begin{array}{l}\text { Acetate, ethanol, DL- } \beta \text {-hydroxybutyrate, glucose, succinate, acrylic acid, cysteic } \\
\text { acid, glycerol, citrate, pyruvate, casamino acids, L-alanine, L-arginine, L-serine, } \\
\text { L-taurine, L-methionine, DMSP and glycine betaine }\end{array}$ & TAS [8] \\
\hline & Energy source & Carbon compounds & \\
\hline & Terminal electron receptor & Oxygen & \\
\hline MIGS-6 & Habitat & Marine & \\
\hline MIGS-6.3 & Salinity & Optimum 100-400 mM & TAS [8] \\
\hline MIGS-22 & Oxygen & Aerobic & TAS [8] \\
\hline MIGS-15 & Biotic relationship & Free-living & TAS [8] \\
\hline MIGS-14 & Pathogenicity & Non-pathogenic & NAS \\
\hline MIGS-4 & Geographic location & Coastal Georgia, USA & TAS [8] \\
\hline MIGS-5 & Sample collection time & June 1996 & NAS \\
\hline MIGS-4.1 MIGS-4.2 & Latitude - Longitude & $31.989616 \mathrm{~N}, 81.022768 \mathrm{~W}$ & NAS \\
\hline MIGS-4.3 & Depth & Surface & NAS \\
\hline MIGS-4.4 & Altitude & Sea level & NAS \\
\hline
\end{tabular}

${ }^{a}$ Evidence codes - TAS: Traceable Author Statement (i.e., a direct report exists in the literature); NAS: Non-traceable Author Statement (i.e., not directly observed for the living, isolated sample, but based on a generally accepted property for the species, or anecdotal evidence).

\section{Table 2 Project information}

\begin{tabular}{lll}
\hline MIGS ID & Property & Term \\
\hline MIGS-31 & Finishing quality & Closed genome [1] \\
MIGS-28 & Libraries used & $1-2 \mathrm{~kb}$ and 12-15 kb inserts [1] \\
MIGS-29 & Sequencing platforms & Sanger \\
MIGS-31.2 & Fold coverage & Not reported \\
MIGS-30 & Assemblers & The TIGR Assembler [20] \\
MIGS-32 & Gene calling method & Glimmer 2.0 [20] \\
& Genome Database release & NCBI Refseq, release version \#8 \\
& Genbank ID & CP000031.2, CP000032.1 \\
& Genbank Date of Release & December 16, 2004 \\
& GOLD ID & Gc00242 \\
& Project relevance & The first heterotrophic marine \\
& & bacterium sequenced \\
\hline
\end{tabular}

Table 3 Genome statistics

\begin{tabular}{|c|c|c|}
\hline Attribute & Value & $\%$ of total ${ }^{\mathrm{a}}$ \\
\hline Genome size (bp) & $4,601,048$ & 100.0 \\
\hline DNA coding region (bp) & $4,144,250$ & 90.1 \\
\hline DNA G + C content (bp) & $2,947,874$ & 64.1 \\
\hline Total genes ${ }^{b}$ & 4371 & 100.0 \\
\hline RNA genes & 64 & 1.5 \\
\hline Protein-coding genes & 4276 & 97.8 \\
\hline
\end{tabular}

The total is based on either the size of the genome in base pairs or the total number of genes in the annotated genome.

${ }^{\mathrm{b}}$ Also includes pseudogenes. 
Table 4 Updates and corrections to the genome sequence

\begin{tabular}{|c|c|c|c|c|}
\hline Accession & Gene locus & CDS & Gene & Type of change \\
\hline YP_166946 & SPO1707a & $\begin{array}{l}\text { Branched-chain amino acid ABC transporter, ATP-binding } \\
\text { protein, putative }\end{array}$ & & Locus name \\
\hline YP_167418 & SPO2192a & $\mathrm{N}$-formylglutamate amidohydrolase & hutG & Locus name \\
\hline YP_165298 & SPO0025 & Hydrolase, NUDIX family & & ORF position \\
\hline YP_165304 & SPO0031 & ErfK/YbiS/YcfS/YnhG family protein & & ORF position \\
\hline YP_165330 & SPO0056 & Hypothetical protein & & ORF position \\
\hline YP_165481 & SPO0212 & Hypothetical protein & & ORF position \\
\hline YP_165606 & SPO0343 & $\begin{array}{l}\text { 2-oxoglutarate dehydrogenase, E2 component, dihydrolipoamide } \\
\text { succinyltransferase }\end{array}$ & $s u c B$ & ORF position \\
\hline YP_165666 & SPO0403 & Conserved domain protein & & ORF position \\
\hline YP_165678 & SPO0415 & D-isomer specific 2-hydroxyacid dehydrogenase family protein & & ORF position \\
\hline YP_165703 & SPO0440 & Thioesterase family protein & & ORF position \\
\hline YP_165709 & SPO0446 & ABC transporter, ATP-binding protein & & ORF position \\
\hline YP_165719 & SPO0456 & Hypothetical protein & & ORF position \\
\hline YP_165753 & SPO0491 & Hypothetical protein & & ORF position \\
\hline YP_165766 & SPO0504 & Hypothetical protein & & ORF position \\
\hline YP_165767 & SPO0505 & Ribosomal protein L15 & rp/O & ORF position \\
\hline YP_165860 & SPO0600 & Carboxynorspermidine decarboxylase & $n s p C$ & ORF position \\
\hline YP_165899 & SPO0644 & Hypothetical protein & & ORF position \\
\hline YP_165937 & SPO0682 & Monooxygenase family protein & & ORF position \\
\hline YP_165950 & SPO0695 & Hypothetical protein & & ORF position \\
\hline YP_008877643 & SPO0876a & Hypothetical protein & & ORF position \\
\hline YP_166130 & SPO0877 & Conserved domain protein & & ORF position \\
\hline YP_166199 & SPO0946 & Phosphomannomutase/phosphoglucomutase & $\operatorname{alg} C$ & ORF position \\
\hline YP_166255 & SPO1003 & ATP-dependent Clp protease, proteolytic subunit ClpP & $c l p P$ & ORF position \\
\hline YP_166256 & SPO1004 & ATP-dependent Clp protease, ATP-binding subunit ClpX & $c l p X$ & ORF position \\
\hline YP_166357 & SPO1106 & Hypothetical protein & & ORF position \\
\hline YP_166419 & SPO1172 & FMN-dependent alpha-hydroxy acid dehydrogenase family protein & & ORF position \\
\hline YP_166421 & SPO1174 & DNA helicase II, putative & & ORF position \\
\hline YP_166518 & SPO1273 & Thymidylate synthase, flavin-dependent & thyx & ORF position \\
\hline YP_166577 & SPO1334 & Hypothetical protein & & ORF position \\
\hline YP_166601 & SPO1359 & Pyruvate, phosphate dikinase & ppdK & ORF position \\
\hline YP_166628 & SPO1386 & HIT family protein & & ORF position \\
\hline YP_166803 & SPO1562 & Glycine cleavage system $T$ protein, putative & & ORF position \\
\hline YP_166874 & SPO1633 & Hypothetical protein & & ORF position \\
\hline YP_167013 & SPO1776 & Pyridine nucleotide-disulphide oxidoreductase family protein & & ORF position \\
\hline YP_167049 & SPO1812 & Adenylate kinase & $a d k-2$ & ORF position \\
\hline YP_167155 & SPO1920 & Tellurite resistance protein & $\operatorname{trg} B$ & ORF position \\
\hline YP_167190 & SPO1955 & Glutaryl-CoA dehydrogenase & gcdH & ORF position \\
\hline YP_167207 & SPO1972 & Nodulation protein $\mathrm{N}$ & & ORF position \\
\hline YP_167208 & SPO1973 & 3-dehydroquinte dehydratase, type II & aroQ & ORF position \\
\hline YP_167281 & SPO2051 & DNA gyrase, A subunit & gyrA & ORF position \\
\hline YP_167284 & SPO2054 & Cytochrome c oxidase assembly protein & & ORF position \\
\hline YP_167368 & SPO2141 & Pyridoxamine 5"-phosphate oxidase, putative & & ORF position \\
\hline YP_167443 & SPO2217 & Excinuclease & & ORF position \\
\hline
\end{tabular}


Table 4 Updates and corrections to the genome sequence (Continued)

\begin{tabular}{|c|c|c|c|c|}
\hline YP_167514 & SPO2290 & Hypothetical protein & & ORF position \\
\hline YP_167549 & SPO2326 & Hypothetical protein & & ORF position \\
\hline YP_167562 & SPO2339 & Enoyl-CoA hydratase/isomerase family protein & & ORF position \\
\hline YP_167570 & SPO2347 & Hypothetical protein & & ORF position \\
\hline YP_167571 & SPO2348 & Sarcosine oxidase, beta subunit family & & ORF position \\
\hline YP_167714 & SPO2499 & Hypothetical protein & & ORF position \\
\hline YP_167808 & SPO2595 & Hypothetical protein & & ORF position \\
\hline YP_167819 & SPO2608 & Aldehyde dehydrogenase, putative & & ORF position \\
\hline YP_167822 & SPO2612 & DNA-binding protein $\mathrm{HU}$, putative & & ORF position \\
\hline YP_008877659 & SPO2723a & Hypothetical protein & & ORF position \\
\hline YP_167934 & SPO2724 & Hypothetical protein & & ORF position \\
\hline YP_167992 & SPO2785 & NADH dehydrogense I, B subunit & nuoB & ORF position \\
\hline YP_168024 & SPO2816 & $\begin{array}{l}\text { Peptide/nickel/opine uptake family } A B C \text { transporter, } \\
\text { permease protein }\end{array}$ & & ORF position \\
\hline YP_168061 & SPO2853 & Cobalt chelatase, CobS subunit & & ORF position \\
\hline YP_168080 & SPO2872 & Cobyrinic acid a,c-diamide synthase & $\operatorname{cob} B$ & ORF position \\
\hline YP_168096 & SPO2888 & Membrane protein, putative & & ORF position \\
\hline YP_168125 & SPO2917 & Glutathione S-transferase family protein & & ORF position \\
\hline YP_168133 & SPO2925 & Sporulation related & & ORF position \\
\hline YP_168143 & SPO2936 & Hypothetical protein & & ORF position \\
\hline YP_168150 & SPO2942 & Hypothetical protein & & ORF position \\
\hline YP_168197 & SPO2991 & Nitroreductase family protein & & ORF position \\
\hline YP_168209 & SPO3003 & AMP-binding enzyme & & ORF position \\
\hline YP_168292 & SPO3089 & ATPase, putative & & ORF position \\
\hline YP_168317 & SPO3114 & Hypothetical protein & & ORF position \\
\hline YP_168354 & SPO3151 & HAD-superfamily subfamily IIA hydrolase, TIGR01459 & & ORF position \\
\hline YP_168406 & SPO3203 & $\begin{array}{l}\text { Guanosine-3',5'-bis(Diphosphate) 3'-pyrophosphohydrolase, } \\
\text { putative }\end{array}$ & & ORF position \\
\hline YP_168423 & SPO3220 & Aminotransferase, classes I and II & & ORF position \\
\hline YP_168448 & SPO3245 & Nicotinate-nucleotide pyrophosphorylase & nadC & ORF position \\
\hline YP_168475 & SPO3278 & Orotidine 5'-phosphate decarboxylase & pyrF & ORF position \\
\hline YP_168540 & SPO3344 & Cys/Met metabolism PLP-dependent enzyme family protein & & ORF position \\
\hline YP_168563 & SPO3367 & Deoxyribose-phosphate aldolase & deoC & ORF position \\
\hline YP_168618 & SPO3422 & ATP-dependent protease La domain protein & & ORF position \\
\hline YP_168712 & SPO3517 & Preprotein translocase, SecE subunit & $\sec E$ & ORF position \\
\hline YP_168722 & SPO3527 & Universal stress protein family protein & & ORF position \\
\hline YP_168735 & SPO3540 & Hypothetical protein & & ORF position \\
\hline YP_168802 & SPO3607 & Hypothetical protein & & ORF position \\
\hline YP_168911 & SPO3717 & Cytosol aminopeptidase family protein & & ORF position \\
\hline YP_168940 & SPO3746 & Adenine deaminse & ade & ORF position \\
\hline YP_169017 & SPO3829 & S-formylglutathione hydrolase, putative & & ORF position \\
\hline YP_169021 & SPO3833 & ATP-dependent RNA helicase, DEAD/DEAH box family & & ORF position \\
\hline YP_164889 & SPOA0058 & Glycine cleavage system protein $\mathrm{H}$ & gcvH-2 & ORF position \\
\hline YP_165979 & SPO0725 & Bacterial SH3 domain family protein & & ORF position, Function \\
\hline YP_167233 & SPO1999 & Collagen domain protein & & ORF position, Function \\
\hline YP_008877641 & SPO0561 & $A B C$ transporter & & Sequence \\
\hline
\end{tabular}


Table 4 Updates and corrections to the genome sequence (Continued)

\begin{tabular}{|c|c|c|c|c|}
\hline YP_008877654 & SPO2024 & Aminotransferase & & Sequence \\
\hline YP_008877662 & SPO3316a & Stress protein & & Sequence \\
\hline YP_008877661 & SPO3904 & Heat shock protein & & Sequence \\
\hline YP_167141 & SPO1905 & Fumarate hydratase, class II & fumc & Sequence, ORF position \\
\hline YP_165491 & SPO0222 & Alanine dehydrogenase & ald & Function \\
\hline YP_165503 & SPO0234 & Lysine dehydrogenase & lysdh & Function \\
\hline YP_165504 & SPO0235 & a-aminoadipic- $\delta$-semialdehyde dehydrogenase & aasadh & Function \\
\hline YP_165716 & SPO0453 & DMSP lyase & $d d d W$ & Function \\
\hline YP_165850 & SPO0590 & Lacl family transcriptional regulator & hps $R$ & Function \\
\hline YP_165851 & SPO0591 & Dihydroxypropanesulfonate (DHPS) TRAP transporter & hpsK & Function \\
\hline YP_165852 & SPO0592 & Dihydroxypropanesulfonate (DHPS) TRAP transporter & hpsL & Function \\
\hline YP_165853 & SPO0593 & Dihydroxypropanesulfonate (DHPS) TRAP transporter & hpsM & Function \\
\hline YP_165854 & SPO0594 & Dihydroxypropanesulfonate-3-dehydrogenase & $h p s N$ & Function \\
\hline YP_165855 & SPO0595 & R or S-dihydroxypropanesulfonate-2-dehydrogenase & hpsO & Function \\
\hline YP_165856 & SPO0596 & S or R-dihydroxypropanesulfonate-2-dehydrogenase & hps $P$ & Function \\
\hline YP_165857 & SPO0597 & UspA stress protein & $h p s Q$ & Function \\
\hline YP_165858 & SPO0598 & Membrane-bound sulfolactate dehydrogenase & $s / C D$ & Function \\
\hline YP_165912 & SPO0657 & Metallochaperone, putative & naat & Function \\
\hline YP_165913 & SPO0658 & $\mathrm{N}$-acetyltaurine amidohydrolase & naas & Function \\
\hline YP_165914 & SPO0659 & LysR family transcriptional regulator & naaR & Function \\
\hline YP_165915 & SPO0660 & $\mathrm{N}$-acetyltaurine $\mathrm{ABC}$ transporter, periplasmic binding protein & naaA & Function \\
\hline YP_165916 & SPO0661 & $\mathrm{N}$-acetyltaurine $\mathrm{ABC}$ transporter, permease protein & $n a a B$ & Function \\
\hline YP_165917 & SPO0662 & $\mathrm{N}$-acetyltaurine $\mathrm{ABC}$ transporter, permease protein & $n a a B^{\prime}$ & Function \\
\hline YP_165918 & SPO0663 & $\mathrm{N}$-acetyltaurine $\mathrm{ABC}$ transporter, ATP-binding protein & naac & Function \\
\hline YP_165919 & SPO0664 & $\mathrm{N}$-acetyltaurine $\mathrm{ABC}$ transporter, ATP-binding protein & naaC' & Function \\
\hline YP_165928 & SPO0673 & Taurine-pyruvate aminotransferase & tpa & Function \\
\hline YP_165929 & SPO0674 & Taurine $A B C$ transporter, periplasmic taurine-binding protein & $\tan A$ & Function \\
\hline YP_165930 & SPO0675 & Taurine ABC transporter, ATP-binding protein & $\tan B$ & Function \\
\hline YP_165931 & SP00676 & Taurine ABC transporter, permease protein & tauc & Function \\
\hline YP_166034 & SPO0781 & Phosphonate $A B C$ transporter substrate-binding protein & phnD & Function \\
\hline YP_166387 & SPO1136 & Diaminobutyric acid transaminase & doeD & Function \\
\hline YP_166388 & SPO1137 & Aspartate-semialdehyde dehydrogenase & doec & Function \\
\hline YP_166389 & SPO1138 & AsnC/Lrp-like DNA-binding protein, transcriptional regulator & doeX & Function \\
\hline YP_166390 & SPO1139 & Na-acetyl-L-2,4-diaminobutyric acid deacetylase & doeb & Function \\
\hline YP_166391 & SPO1140 & Ectoine hydrolase & doeA & Function \\
\hline YP_166392 & SPO1141 & Ectoine utilization protein EutC & eutC & Function \\
\hline YP_166394 & SPO1143 & Ectoine utilization protein EutA & eutA & Function \\
\hline YP_166396 & SPO1145 & $\begin{array}{l}\text { Ectoine/5-hydroxyectoine TRAP transporter, periplasmic } \\
\text { binding protein }\end{array}$ & uehC & Function \\
\hline YP_166397 & SPO1146 & $\begin{array}{l}\text { Ectoine/5-hydroxyectoine TRAP transporter, small integral } \\
\text { membrane protein }\end{array}$ & uehB & Function \\
\hline YP_166398 & SPO1147 & $\begin{array}{l}\text { Ectoine/5-hydroxyectoine TRAP transporter, large integral } \\
\text { membrane protein }\end{array}$ & uehA & Function \\
\hline YP_166399 & SPO1148 & Transcriptional regulator, GntR family & gntR & Function \\
\hline YP_166792 & SPO1551 & Trimethylamine (TMA) monooxygenase & $t m m$ & Function \\
\hline YP_166837 & SPO1596 & DMSP lyase & $d d d Q$ & Function \\
\hline
\end{tabular}


Table 4 Updates and corrections to the genome sequence (Continued)

\begin{tabular}{|c|c|c|c|c|}
\hline YP_166942 & SPO1703 & DMSP lyase & $d d d D$ & Function \\
\hline YP_167149 & SPO1914 & NADPH-dependent acrylyl-CoA reductase & acul & Function \\
\hline YP_167183 & SPO1948 & Phosphate $A B C$ transporter substrate-binding protein & psts & Function \\
\hline YP_167275 & SPO2045 & 3-methylmercaptopropionyl-CoA ligase & $d m d B$ & Function \\
\hline YP_167522 & SPO2299 & DMSP lyase & $d d d P$ & Function \\
\hline YP_167578 & SPO2355 & Isethionate dissimilation regulator & isen & Function \\
\hline YP_167579 & SPO2356 & Isethionate TRAP transporter & isem & Function \\
\hline YP_167580 & SPO2357 & Isethionate TRAP transporter & isel & Function \\
\hline YP_167581 & SPO2358 & Isethionate TRAP transporter & isek & Function \\
\hline YP_167582 & SPO2359 & Isethionate dehydrogenase & isel & Function \\
\hline YP_167694 & SPO2477 & Manganese uptake regulator & mur & Function \\
\hline YP_168390 & SPO3187 & (2R)-3-sulfolactate dehydrogenase & $\mathrm{comC}$ & Function \\
\hline YP_168503 & SPO3307 & Lysine-ketoglutarate reductase & Ikr & Function \\
\hline YP_168559 & SPO3363 & Manganese $A B C$ transporter, permease protein & sitD & Function \\
\hline YP_168560 & SPO3364 & Manganese $A B C$ transporter, permease protein & sitC & Function \\
\hline YP_168561 & SPO3365 & Manganese $A B C$ transporter, ATP-binding protein & sitB & Function \\
\hline YP_168562 & SPO3366 & Manganese $A B C$ transporter, periplasmic protein & sitA & Function \\
\hline YP_168752 & SPO3557 & $\begin{array}{l}\text { Sulfite dehydrogenase subunit SoeC; transmembrane } \\
\text { sulfate transporter }\end{array}$ & soec & Function \\
\hline YP_168753 & SPO3558 & $\begin{array}{l}\text { Sulfite dehydrogenase iron-sulfur cluster-binding } \\
\text { subunit SoeB; cytosolic protein }\end{array}$ & soeB & Function \\
\hline YP_168754 & SPO3559 & $\begin{array}{l}\text { Sulfite dehydrogenase molybdopterin cofactor-binding } \\
\text { subunit SoeA; cytosolic protein }\end{array}$ & soeA & Function \\
\hline YP_168755 & SPO3560 & Phosphate acetyltransferase & pta & Function \\
\hline YP_168757 & SPO3562 & Taurine transcriptional regulator & $\operatorname{tau} R$ & Function \\
\hline YP_168992 & SPO3804 & 3-methylmercaptopropionyl-CoA dehydrogenase & $d m d C$ & Function \\
\hline YP_168993 & SPO3805 & Methylthioacryloyl-CoA hydratase & $d m d D$ & Function \\
\hline YP_164988 & SPOA0157 & Sulfite exporter & cuyZ & Function \\
\hline YP_164989 & SPOA0158 & L-cysteate sulfo-lyase & CuyA & Function \\
\hline YP_164990 & SPOA0159 & Transcriptional regulator cuyR & cuy $R$ & Function \\
\hline YP_165136 & SPOA0309 & Sulphoacetaldehyde acetyltransferase & & Function \\
\hline YP_008877636 & SPO0344a & Hypothetical protein & & New ORF \\
\hline YP_008877637 & SPO0346a & Hypothetical protein & & New ORF \\
\hline YP_008877638 & SPO0360a & Hypothetical protein & & New ORF \\
\hline YP_008877639 & SPO0491a & Hypothetical protein & & New ORF \\
\hline YP_008877640 & SPO0504a & Hypothetical protein & & New ORF \\
\hline YP_008877642 & SPO0628a & Hypothetical protein & & New ORF \\
\hline YP_008877644 & SPO1044a & Hypothetical protein & & New ORF \\
\hline YP_008877645 & SPO1094a & Hypothetical protein & & New ORF \\
\hline YP_008877646 & SPO1226a & Hypothetical protein & & New ORF \\
\hline YP_008877647 & SPO1252a & Transcriptional regulator & & New ORF \\
\hline YP_008877648 & SPO1310a & Hypothetical protein & & New ORF \\
\hline YP_008877649 & SPO1337a & Hypothetical protein & & New ORF \\
\hline YP_008877650 & SPO1352a & Hypothetical protein & & New ORF \\
\hline YP_008877651 & SPO1356a & Signal transduction & & New ORF \\
\hline YP_008877652 & SPO1364a & Hypothetical protein & & New ORF \\
\hline
\end{tabular}


Table 4 Updates and corrections to the genome sequence (Continued)

\begin{tabular}{llll}
\hline YP_008877653 & SPO1412a & Hypothetical protein & New ORF \\
YP_008877655 & SPO2213a & Hypothetical protein & New ORF \\
YP_008877656 & SPO2341a & Hypothetical protein & New ORF \\
YP_008877657 & SPO2478 & RNA helicase & New ORF \\
YP_008877658 & SPO2652a & Polyketide cyclase & New ORF \\
YP_008877660 & SPO2973a & Hypothetical protein & New ORF \\
YP_008877663 & SPO3452a & Hypothetical protein & New ORF \\
YP_008877664 & SPO3498a & Hypothetical protein & New ORF \\
YP_008877665 & SPO3673a & Hypothetical protein & New ORF \\
AHC32567 & SPOA0087a & Esterase-lipase & New ORF \\
AHC32568 & SPOA0272a & Hypothetical protein & New ORF \\
YP_165305 & - & Hypothetical protein & Removed ORF \\
YP_165605 & - & Hypothetical protein & Removed ORF \\
YP_166669 & - & Hypothetical protein & Removed ORF \\
YP_168865 & - & Hypothetical protein & Removed ORF \\
YP_165238 & - & Hypothetical protein & Removed ORF \\
\hline
\end{tabular}

Members of the marine Roseobacter lineage are capable of oxidizing sulfite and thiosulfate [34,35], and the genome sequence of $R$. pomeroyi DSS-3 revealed the sox gene cluster that mediates these processes [1,4]. Recently, the reverse dissimilatory sulfite reductase gene cluster was found in sediment-dwelling roseobacters, and homologs to the sulfite reductase genes from this pathway (soeABC) were identified in the $R$. pomeroyi DSS-3 genome [36]. R. Pomeroyi DSS-3 was initially studied as a member of an ecologically important bacterial taxon that appeared unusually amenable to cultivation [5], but has now played a major role in improving our understanding of global sulfur transformations.

Studies of the $R$. pomeroyi DSS-3 genome have also provided a better understanding of the genes involved in processing organic nitrogen compounds, such as taurine and $\mathrm{N}$-acetyltaurine $[24,31,32]$. The bacterium can catabolize lysine by using the saccharopine pathway, which is used by many plants and animals, or by using the lysine dehydrogenase pathway. Under high salt conditions, it preferentially uses the latter pathway, leading to biosynthesis of the osmolyte aminoadipate. The function of several genes in both lysine pathways has recently been experimentally verified [37].

R. pomeroyi DSS-3 genome hosts at least 28 tripartite ATP-independent periplasmic (TRAP) transporters [1]. While the substrates for many of these transporters are not yet known, the TRAP transporters responsible for the uptake of 2,3-dihydroxypropane-1-sufonate (hpsKLM) [29], isethionate (iseKLM) [25], and ectoine and hydroxyectoine have been characterized (uehABC) [38,39]. Ectoine and hydroxyectoine are used as compatible solutes by some bacteria and phytoplankton, although $R$. pomeroyi DSS-3 can also assimilate carbon and nitrogen from them [39]. Several genes involved in ectoine metabolism (doe, eut, ueh) have been found in the $R$. pomeroyi DSS-3 genome based on homology with genes in Halomonas elongata DSM $2581 \mathrm{~T}$ [40].

Progress has been made in understanding the mechanisms of metal uptake in $R$. pomeroyi DSS-3. The manganese uptake regulator mur has been experimentally validated, as have the $\mathrm{ABC}$ transporter genes for manganese metabolism (sitABCD) [41]. In total, 69 annotation changes were made based on new experimental data identifying genes responsible for carbon, nitrogen, sulfur, and metal uptake and metabolism [42].

Proteomics [42] and mRNA sequencing have resulted in 120 protein coding regions being identified, removed or corrected in the updated genome. A detailed proteomic study of $R$. pomeroyi DSS-3 under diverse growth conditions resulted in the identification of 26 novel open reading frames (ORFs) and 5 sequencing errors [42]. The function of most of the new genes is not known and 16 of the expressed polypeptides do not have known homologs. The 26 ORFs missed in the original annotation is a significant number but less than the $1 \%$ error rate predicted for Glimmer 2 [20]. The proteomic analysis was also able to correct the start sites of 64 genes [42], enhancing the information that had been obtained only from the DNA sequence [20]. Many of the ORFs identified by proteomics were independently confirmed using strand-specific messenger RNA sequences from continuous cultures [43] and the gene calling software Glimmer 3 [44]. This method also identified several genes that were originally annotated in the wrong orientation, including a novel bacterial collagen gene (SPO1999). 
A list of genome updates based on these biochemical, genetic, and -omics approaches is provided in Table 4, and full details in Additional file 1: Table S1. The updated annotations have been incorporated into the official genome record at the National Center for Biotechnology Information (Bethesda, MD, USA) under accession numbers CP000031.2 and CP000032.1 and Roseobase (http://roseobase.org).

\section{Conclusion}

Ten years after the publication of the Ruegeria pomeroyi DSS-3 genome sequence, advances in knowledge of gene function and structural genome features motivated an annotation update. As an ecologically-relevant heterotrophic marine bacterium that is amenable to laboratory studies and genetic manipulation, $R$. pomeroyi is serving as a valuable model organism for investigations of the ecology, biochemistry, and biogeochemistry of ocean microbes.

\section{Additional file}

Additional file 1: Table S1. Full details of updates and corrections to the Ruegeria pomeroyi DSS-3 genome sequence.

\section{Competing interests}

The authors declare that they have no competing interests.

\section{Authors' contributions}

ARR conceived of the study, carried out the bioinformatics analyses, and wrote the manuscript. CBS carried out the bioinformatics analyses and wrote the manuscript. MAM conceived of the study and wrote the manuscript. All authors read and approved the final manuscript.

\section{Acknowledgements}

This work was supported by NSF grant MCB-1158037 and the Gordon and Betty Moore Foundation. We are grateful to A. Cook and K. Denger for adopting R. pomeroyi DSS-3 in their sulfonate research, and A. Burns for reviewing the mRNA data.

Received: 12 June 2014 Accepted: 16 June 2014

Published: 8 December 2014

\section{References}

1. Moran MA, Buchan A, González JM, Heidelberg JF, Whitman WB, Kiene RP, Henriksen JR, King GM, Belas R, Fuqua C, Brinkac L, Lewis M, Shivani J, Weaver B, Pai G, Eisen JA, Rahe E, Sheldon WM, Ye W, Miller TR, Carlton J, Rasko DA, Paulsen IT, Ren Q, Daugherty SC, Deboy RT, Dodson RJ, Durkin AS, Madupu R, Nelson WC, et al. Genome sequence of Silicibacter pomeroyi reveals adaptations to the marine environment. Nature. 2004; 432:910-3. PubMed http://dx.doi.org/10.1038/nature03170.

2. Reisch CR, Stoudemayer MJ, Varaljay VA, Amster IJ, Moran MA, Whitman WB. Novel pathway for assimilation of dimethylsulphoniopropionate widespread in marine bacteria. Nature. 2011; 473:208-11. PubMed http:// dx.doi.org/10.1038/nature10078

3. Moran MA, Reisch CR, Kiene RP, Whitman WB. Genomic insights into bacterial DMSP transformations. Annu Rev Mar Sci. 2012; 4:523-42. PubMed http://dx.doi.org/10.1146/annurev-marine-120710-100827.

4. Newton RJ, Griffin LE, Bowles KM, Meile C, Gifford S, Givens CE, Howard EC, King E, Oakley CA, Reisch CR, Rinta-Kanto JM, Sharma S, Sun S, Varaljay V, Vila-Costa M, Westrich JR, Moran MA. Genome characteristics of a generalist marine bacterial lineage. ISME J. 2010; 4:784-98. PubMed http:// dx.doi.org/10.1038/ismej.2009.150.

5. González JM, Moran MA. Numerical dominance of a group of marine bacteria in the alpha-subclass of the class Proteobacteria in coastal seawater. Appl Environ Microbiol. 1997; 63:4237-42. PubMed.
6. Buchan A, González JM, Moran MA. Overview of the marine roseobacter lineage. App/ Environ Microbiol. 2005; 71:5665-77. PubMed http://dx.doi.org/ 10.1128/AEM.71.10.5665-5677.2005.

7. Yi H, Lim YW, Chun J. Taxonomic evaluation of the genera Ruegeria and Silicibacter: a proposal to transfer the genus Silicibacter Petursdottir and Kristjansson 1999 to the genus Ruegeria Uchino et al. 1999. Int I Syst Evol Microbiol. 2007; 57:815-9. PubMed http://dx.doi.org/10.1099/ijs.0.64568-0.

8. González JM, Covert JS, Whitman WB, Henriksen JR, Mayer F, Scharf B, Schmitt R, Buchan A, Fuhrman JA, Kiene RP, Moran MA. Silicibacter pomeroyi sp. nov. and Roseovarius nubinhibens sp. nov., dimethylsulfoniopropionate-demethylating bacteria from marine environments. Int J Syst Evol Microbiol. 2003; 53:1261-9. PubMed http://dx. doi.org/10.1099/ijs.0.02491-0.

9. Field D, Garrity G, Gray T, Morrison N, Selengut J, Sterk P, Tatusova T, Thomson N, Allen MJ, Angiuoli SV, Stevens R, Swift P, Taylor C, Tateno Y, Tett A, Turner S, Ussery D, Vaughan B, Ward N, Whetzel T, San Gl, Wilson G, Wipat A. The minimum information about a genome sequence (MIGS) specification. Nat Biotechnol. 2008; 26:541-7. PubMed http://dx.doi.org/10.1038/nbt1360.

10. Woese CR, Kandler $\mathrm{O}$, Wheelis ML. Towards a natural system of organisms: proposal for the domains Archaea, Bacteria, and Eucarya. Proc Natl Acad Sci USA. 1990; 87:4576-9. PubMed http://dx.doi.org/10.1073/pnas.87.12.4576.

11. Garrity GM, Bell JA, Lilburn TPhylum XIV. Proteobacteria phyl. nov. In: Garrity GM, Brenner DJ, Krieg NR, Staley JT, editors. Bergey's Manual of Systematic Bacteriology, Volume 2. 2nd ed. New York: Part B, Springer; 2005: p. 1.

12. Validation List No. 107. List of new names and new combinations previously effectively, but not validly, published. Int J Syst Evol Microbiol. 2006; 56:1-6. PubMed http://dx.doi.org/10.1099/ijs.0.64188-0.

13. Garrity GM, Bell JA, Class LT I. Alphaproteobacteria class. nov. In: Garrity GM, Brenner DJ, Krieg NR, Staley JT, editors. Bergey's Manual of Systematic Bacteriology, Volume 2. 2nd ed. New York: Part C, Springer; 2005: p. 1.

14. Garrity GM, Bell JA, Order LT III. Rhodobacterales ord. nov. In: Garrity GM, Brenner DJ, Krieg NR, Staley JT, editors. Bergey's Manual of Systematic Bacteriology, Volume 2. 2nd ed. New York: Part C, Springer; 2005: p. 161.

15. Garrity GM, Bell JA, Family LT I. Rhodobacteraceae fam. nov. In: Garrity GM, Brenner DJ, Krieg NR, Staley JT, editors. Bergey's Manual of Systematic Bacteriology, Volume 2. 2nd ed. New York: Part C, Springer; 2005: p. 161.

16. Validation List no. 68. Validation of publication of new names and new combinations previously effectively published outside the IJSB. Int J Syst Bacteriol. 1999; 49:1-3. http://dx.doi.org/10.1099/00207713-49-1-1.

17. Uchino Y, Hirata A, Yokota A, Sugiyama J. Reclassification of marine Agrobacterium species: Proposals of Stappia stellulata gen. nov., comb. nov., Stappia aggregata sp. nov., nom. rev., Ruegeria atlantica gen. nov., comb. nov., Ruegeria gelatinovora comb. nov., Ruegeria algicola comb. nov., and Ahrensia kieliense gen. nov., sp. nov., nom. rev. J Gen Appl Microbiol. 1998; 44:201-10. PubMed http://dx.doi.org/10.2323/jgam.44.201.

18. Martens T, Heidorn T, Pukall R, Simon M, Tindall BJ, Brinkhoff T. Reclassification of Roseobacter gallaeciensis Ruiz-Ponte et al. 1998 as Phaeobacter gallaeciensis gen. nov., comb. nov., description of Phaeobacter inhibens sp. nov., reclassification of Ruegeria algicola (Lafay et al. 1995) Uchino et al. 1999 as Marinovum algicola gen. nov., comb. nov., and emended descriptions of the genera Roseobacter, Ruegeria and Leisingera. Int J Syst Evol Microbiol. 2006; 56:1293-304. PubMed http://dx. doi.org/10.1099/ijs.0.63724-0.

19. Vandecandelaere I, Nercessian O, Segaert E, Achouak W, Faimali M, Vandamme P. Ruegeria scottomollicae sp. nov., isolated from a marine electroactive biofilm. Int J Syst Evol Microbiol. 2008; 58:2726-33. PubMed http://dx.doi.org/10.1099/ijs.0.65843-0.

20. Delcher AL. Improved microbial gene identification with GLIMMER. Nucleic Acids Res. 1999; 27:4636-41. PubMed http://dx.doi.org/10.1093/nar/27.23.4636

21. Eisen JA, Nelson KE, Paulsen IT, Heidelberg JF, Wu M, Dodson RJ, Deboy R, Gwinn ML, Nelson WC, Haft DH, Hickey EK, Peterson JD, Durkin AS, Kolonay J, Yang F, Holt I, Umayam LA, Mason T, Brenner M, Shea TP, Parksey D, Nierman WC, Feldblyum TV, Hansen CL, Craven MB, Radune D, Vamathevan J, Khouri H, White O, Gruber TM, et al. The complete genome sequence of Chlorobium tepidum TLS, a photosynthetic, anaerobic, green-sulfur bacterium. Proc Natl Acad Sci USA. 2002; 99:9509-14. PubMed http://dx.doi.org/10.1073/ pnas.132181499.

22. Howard EC, Henriksen JR, Buchan A, Reisch CR, Bürgmann H, Welsh R, Ye W, González JM, Mace K, Joye SB, Kiene RP, Whitman WB, Moran MA. Bacterial taxa that limit sulfur flux from the ocean. Science. 2006; 314:649-52. PubMed http://dx.doi.org/10.1126/science.1130657. 
23. Todd JD, Curson ARJ, Kirkwood M, Sullivan MJ, Green RT, Johnston AWB. DddQ, a novel, cupin-containing, dimethylsulfoniopropionate lyase in marine roseobacters and in uncultured marine bacteria. Environ Microbiol. 2011; 13:427-38. PubMed http://dx.doi.org/10.1111/j.1462-2920.2010.02348.x.

24. Gorzynska AK, Denger K, Cook AM, Smits THM. Inducible transcription of genes involved in taurine uptake and dissimilation by Silicibacter pomeroyi DSS-3. Arch Microbiol. 2006; 185:402-6. PubMed http://dx.doi.org/10.1007/ s00203-006-0106-8.

25. Weinitschke S, Sharma PI, Stingl U, Cook AM, Smits THM. Gene clusters involved in isethionate degradation by terrestrial and marine bacteria. Appl Environ Microbiol. 2010; 76:618-21. PubMed http://dx.doi.org/10.1128/ AEM.01818-09.

26. Todd JD, Kirkwood M, Newton-Payne S, Johnston AWB. DddW, a third DMSP lyase in a model Roseobacter marine bacterium, Ruegeria pomeroyi DSS-3. ISME J. 2012; 6:223-6. PubMed http://dx.doi.org/10.1038/ismej.2011.79.

27. Todd JD, Curson ARJ, Sullivan MJ, Kirkwood M, Johnston AWB. The Ruegeria pomeroyi acul gene has a role in DMSP catabolism and resembles yhdH of $E$. coli and other bacteria in conferring resistance to acrylate. PLOS ONE. 2012; 7:e35947. PubMed http://dx.doi.org/10.1371/journal. pone.0035947

28. Varaljay VA, Howard EC, Sun S, Moran MA. Deep sequencing of a dimethylsulfoniopropionate-degrading gene $(d m d A)$ by using PCR primer pairs designed on the basis of marine metagenomic data. App/ Environ Microbiol. 2010; 76:609-17. PubMed http://dx.doi.org/10.1128/ AEM.01258-09.

29. Mayer J, Huhn T, Habeck M, Denger K, Hollemeyer K, Cook AM. 2,3-Dihydroxypropane-1-sulfonate degraded by Cupriavidus pinatubonensis JMP134: purification of dihydroxypropanesulfonate 3-dehydrogenase. Microbiology. 2010; 156:1556-64. PubMed http://dx.doi.org/10.1099/ mic.0.037580-0.

30. Denger K, Smits THM, Cook AM. L-cysteate sulpho-lyase, a widespread pyridoxal 5'-phosphate-coupled desulphonative enzyme purified from Silicibacter pomeroyi DSS-3. Biochem J. 2006; 394:657-64. PubMed http:// dx.doi.org/10.1042/BJ20051311.

31. Denger K, Lehmann S, Cook AM. Molecular genetics and biochemistry of $\mathrm{N}$-acetyltaurine degradation by Cupriavidus necator H16. Microbiology. 2011; 157:2983-91. PubMed http://dx.doi.org/10.1099/mic.0.048462-0.

32. Cook AM, Denger K. Metabolism of taurine in microorganisms: a primer in molecular biodiversity? Adv Exp Med Biol. 2006; 583:3-13. PubMed http://dx.doi.org/10.1007/978-0-387-33504-9_1.

33. Denger K, Mayer J, Buhmann M, Weinitschke S, Smits THM, Cook AM. Bifurcated degradative pathway of 3-sulfolactate in Roseovarius nubinhibens ISM via sulfoacetaldehyde acetyltransferase and (S)-cysteate sulfolyase. J Bacteriol. 2009; 191:5648-56. PubMed http://dx.doi.org/ 10.1128/JB.00569-09.

34. González JM, Kiene RP, Moran MA. Transformation of sulfur compounds by an abundant lineage of marine bacteria in the alpha-subclass of the class Proteobacteria. Appl Environ Microbiol. 1999; 65:3810-9. PubMed.

35. Moran MA, González JM, Kiene RP. Linking a bacterial taxon to sulfur cycling in the sea: Studies of the marine Roseobacter group. Geomicrobiol J. 2003; 20:375-88. http://dx.doi.org/10.1080/01490450303901.

36. Lehmann S. Sulfite dehydrogenases in organotrophic bacteria: enzymes, genes and regulation. University of Konstanz: Doctoral Dissertation; 2013: p. 142

37. Neshich IA, Kiyota E, Arruda P. Genome-wide analysis of lysine catabolism in bacteria reveals new connections with osmotic stress resistance. ISME J. 2013; 7:2400-10. PubMed http://dx.doi.org/10.1038/ismej.2013.123.

38. Mulligan C, Fischer M, Thomas GH. Tripartite ATP-independent periplasmic (TRAP) transporters in Bacteria and Archaea. FEMS Microbiol Rev. 2011; 35:68-86. PubMed http://dx.doi.org/10.1111/j.1574-6976.2010.00236.x.

39. Lecher J, Pittelkow M, Zobel S, Bursy J, Bönig T, Smits SH, Schmitt L, Bremer E. The crystal structure of UehA in complex with ectoine-A comparison with other TRAP-T binding proteins. J Mol Biol. 2009; 389:58-73. PubMed http://dx.doi.org/10.1016/j.jmb.2009.03.077.

40. Schwibbert K, Marin-Sanguino A, Bagyan I, Heidrich G, Lentzen G, Seitz H, Rampp M, Schuster SC, Klenk HP, Pfeiffer F, Oesterhelt D, Kunte HJ. A blueprint of ectoine metabolism from the genome of the industrial producer Halomonas elongata DSM 2581 T. Environ Microbiol. 2011; 13:1973-94. PubMed http://dx.doi.org/10.1111/j.1462-2920.2010.02336.x.
41. Green RT, Todd JD, Johnston AWB. Manganese uptake in marine bacteria; the novel MntX transporter is widespread in Roseobacters, Vibrios, Alteromonadales and the SAR11 and SAR116 clades. ISME J. 2013; 7:581-91. PubMed http://dx.doi.org/10.1038/ismej.2012.140.

42. Christie-Oleza JA, Miotello G, Armengaud J. High-throughput proteogenomics of Ruegeria pomeroyi: seeding a better genomic annotation for the whole marine Roseobacter clade. BMC Genomics. 2012; 13:73. PubMed http://dx.doi.org/10.1186/1471-2164-13-73.

43. Chan LK, Newton RJ, Sharma S, Smith CB, Rayapati P, Limardo AJ, Meile C, Moran MA. Transcriptional changes underlying elemental stoichiometry shifts in a marine heterotrophic bacterium. Front Microbiol. 2012; 3:159. PubMed http://dx.doi.org/10.3389/fmicb.2012.00159.

44. Delcher AL, Bratke KA, Powers EC, Salzberg SL. Identifying bacterial genes and endosymbiont DNA with Glimmer. Bioinformatics. 2007; 23:673-9. PubMed http://dx.doi.org/10.1093/bioinformatics/btm009.

doi:10.1186/1944-3277-9-11

Cite this article as: Rivers et al:: An Updated genome annotation for the model marine bacterium Ruegeria pomeroyi DSS-3. Standards in Genomic Sciences 2014 9:11.

\section{Submit your next manuscript to BioMed Central and take full advantage of:}

- Convenient online submission

- Thorough peer review

- No space constraints or color figure charges

- Immediate publication on acceptance

- Inclusion in PubMed, CAS, Scopus and Google Scholar

- Research which is freely available for redistribution 